\title{
Ionization by few-cycle pulses: Tracing the electron orbits
}

\author{
D. B. Milošević, ${ }^{1}$ G. G. Paulus, ${ }^{2, *}$ and W. Becker ${ }^{3}$ \\ ${ }^{1}$ Faculty of Science, University of Sarajevo, Zmaja od Bosne 35, 71000 Sarajevo, Bosnia and Herzegovina \\ ${ }^{2}$ Department of Physics, Texas A\&M University, College Station, Texas 77843-4242, USA \\ ${ }^{3}$ Max-Born-Institut, Max-Born-Strasse 2a, 12489 Berlin, Germany
}

(Received 28 August 2004; published 14 June 2005)

\begin{abstract}
High-order above-threshold ionization by few-cycle laser pulses is analyzed in terms of quantum orbits. For a given carrier-envelope phase, the number of contributing orbits and their ionization and rescattering times determine the shape of the angle-resolved spectrum in all detail. Conversely, analysis of a given spectrum reveals the carrier-envelope phase and the various interfering pathways from which the electron could choose.
\end{abstract}

DOI: 10.1103/PhysRevA.71.061404

PACS number(s): 32.80.Rm, 42.50.Hz, 42.65.Re

Few-cycle pulses in the near infrared with stable and controllable carrier-envelope (CE) phase have come to provide a powerful tool for the investigation of the atomic ionization dynamics, which is much more sensitive and versatile than the long pulses utilized thus far [1]. Varying the CE phase, one can probe the atom in many different ways and extract much more information than can be gained with a long pulse whose shape is identical from cycle to cycle [2-6].

In the high-intensity low-frequency regime where most intense-laser-atom experiments have been carried out, our physical understanding relies on the rescattering model [7]. For a specified final state, this model yields an ionization time $t_{0}$, i.e., the time when the electron enters the continuum via ionization, and a rescattering time $t_{1}$, when the oscillating laser field drives the electron back to its parent ion, off which in the case of high-order above-threshold ionization (HATI), it rescatters into its final state. For long pulses, these two times are rigidly connected both with respect to each other and to their location within the laser cycle, and the ionization dynamics within each cycle of the pulse are identical (provided depletion of the ground state can be neglected). For few-cycle pulses, this is no longer so, and the additional freedom is reflected in the HATI electron spectrum $[5,8]$.

Laser-atom physics in the high-intensity low-frequency regime combines in a unique fashion quantum-mechanical and classical features, viz. tunneling and interference of matter versus classical propagation. An appealing and efficient description of both aspects is afforded by the method of "quantum orbits" $[9,10]$, which relies upon a saddle-point evaluation of the quantum-mechanical transition amplitude and retrieves Feynman's path integral in this context [11]. This method has been successfully applied to various laseratom processes in the presence of an infinitely long pulse [12]. It can be employed for few-cycle pulses as well [6]. In this paper, we will utilize it to analyze HATI by few-cycle pulses. We concentrate on high-order above-threshold ionization (ATI), since this is much more sensitive to the CE phase than ATI of low order (direct electrons) [5].

For low laser frequency and high intensity, the strong-

\footnotetext{
*Also at Ludwig-Maximilians-Universität München and MaxPlanck-Institut für Quantenoptik, 85748 Garching, Germany.
}

field approximation (SFA) applies, which neglects the effect of the laser field on the initial bound state $|0\rangle$ and the effect of the binding potential on the final continuum state $|\mathbf{p}\rangle$ with momentum $\mathbf{p}$. The transition amplitude from the former to the latter state can be conveniently evaluated in the saddlepoint approximation, which returns it in the form [10-12]

$$
M_{\mathbf{p} 0}=\sum_{s} a_{s} \exp \left[i S_{s}(\mathbf{p})\right]
$$

Here the $a_{s}$ are complex coefficients whose specific form does not concern us here and

$$
\begin{aligned}
S_{s}(\mathbf{p})= & -\frac{1}{2} \int_{t_{1 s}}^{\infty} d \tau[\mathbf{p}+\mathbf{A}(\tau)]^{2}-\frac{1}{2} \int_{t_{0 s}}^{t_{1 s}} d \tau\left[\mathbf{k}_{s}+\mathbf{A}(\tau)\right]^{2} \\
& +I_{p} t_{0 s}(s=1,2, \ldots)
\end{aligned}
$$

is the action of an electron along its path from the ground state of the atom with ionization potential $I_{p}$ to the detector. This path starts when the electron is born by ionization at the instant of time $t_{0 s}$ with drift momentum $\mathbf{k}_{s}$. It rescatters off the ion at the later time $t_{1 s}$, such that it reaches the detector with momentum p. For given $\mathbf{p}$, there are several such paths or orbits, which we number by the subscript $s$. They are obtained as (complex) solutions of the so-called saddle-point equations [10-12]. Any such solution $\left(t_{0 s}, t_{1 s}, \mathbf{k}_{s}\right)$ defines the electron's (complex) "quantum orbit" [12]

$$
\mathbf{x}(t)= \begin{cases}\left(t-t_{0 s}\right) \mathbf{k}_{s}+\int_{t_{0 s}}^{t} d \tau \mathbf{A}(\tau) & \left(t_{0 s \mathrm{R}} \leqslant t \leqslant t_{1 s \mathrm{R}}\right), \\ \left(t-t_{1 s}\right) \mathbf{p}+\int_{t_{1 s}}^{t} d \tau \mathbf{A}(\tau) \quad\left(t \geqslant t_{1 s \mathrm{R}}\right),\end{cases}
$$

where $t_{x \mathrm{R}} \equiv \operatorname{Re} t_{x}$. While $\mathbf{x}\left(t_{0 s}\right)=\mathbf{0}$, the real part of the orbit starts at $\mathbf{x}\left(\operatorname{Re} t_{0 s}\right) \neq \mathbf{0}$. Examples of such orbits are presented below in Fig. 3.

Conceptually, the method of quantum orbits is very straightforward: the solutions of the saddle-point equations have to be determined and inserted into Eq. (1). However, finding the solutions is not always a trivial task, in particular for a few-cycle pulse. Details of the procedure we employed will be published elsewhere.

For a laser field $\mathbf{A}(t)$ that is periodic with the period $T$ 
$=2 \pi / \omega$, with any solution $\left(t_{0}, t_{1}, \mathbf{k}\right)$ of the saddle-point equations, $\left(t_{0}+T, t_{1}+T, \mathbf{k}\right)$ will be another solution, that is, the quantum orbits are identical from one cycle to the next. Together with the form (2) of the action, this has the consequence that $\left(U_{p}\right.$ denotes the ponderomotive energy of the infinitely extended laser field and $E_{\mathbf{p}} \equiv \mathbf{p}^{2} / 2$ )

$$
M_{\mathbf{p} 0}=\sum_{n} \delta\left(E_{\mathbf{p}}+I_{p}+U_{p}-n \omega\right) \sum_{s^{\prime}} a_{s^{\prime}} e^{i S_{s^{\prime}}(\mathbf{p})},
$$

where the sum over $s^{\prime}$ is now restricted to quantum orbits having return times $t_{1 s^{\prime}}$ within only one cycle of the field, say $0 \leqslant t_{1 s^{\prime} \mathrm{R}}<T$. Hence, periodicity of the laser field implies a spectrum having discrete peaks spaced by $\omega$. In other words, all energies other than those allowed by the energyconserving $\delta$ function in Eq. (4) are eliminated by destructive interference of the contributions from the various cycles of the field.

We will consider the linearly polarized few-cycle pulse with the electric-field vector

$$
\mathbf{E}(t)=E_{0}(t) \cos (\omega t+\phi) \hat{\mathbf{e}}
$$

for $0 \leqslant t \leqslant T_{p}=n_{p} T$ with integer $n_{p}$, and zero outside this interval. The sine-square envelope function $E_{0}(t)$ $=E_{0} \sin ^{2}\left(\omega t / 2 n_{p}\right)$ assumes its maximum at $t=T_{p} / 2$. The $\mathrm{CE}$ phase $\phi$ specifies the delay between this maximum of the envelope and the nearest maximum of the electric field of the carrier wave with frequency $\omega$. Carrier-envelope phases $\phi$ $=0$ and $\phi=\pi / 2$ (modulo $\pi$ ) correspond to cosine and sine pulses with respect to the maximum of the envelope. For pulses this short, the quantum orbits are different from cycle to cycle and, for each cycle, have to be calculated separately. We can no longer use Eq. (4), but have to return to Eq. (1), which receives contributions from all orbits with $0<t_{1 s \mathrm{R}}$ $<\infty$ including times when the pulse has already passed through.

Therefore, the number of orbits that, in principle, need be considered is much larger than in the periodic case. However, only those orbits make significant contributions, for which the electric field at the ionization time is sufficiently strong. In addition, for the high-energy part of the electron spectrum, the electric field after the rescattering time must still be sufficiently strong, since it is the latter field that accelerates electrons to energies higher than $\gtrsim 4 U_{p}$. These two conditions combined entail that for pulses with $T_{p} \lesssim 8 T$ only orbits contribute that start and rescatter close to the maximum of the field envelope. Normally, there are no more than two pairs of such orbits, whose rescattering times $t_{1 s}$ are approximately one cycle apart.

We will now present examples of the effects characteristic of HATI with few-cycle pulses. We assume the field (5) for $n_{p}=7$, which corresponds to 6.8 fs full width at half maximum in intensity. The rescattering potential is modeled by a potential of the Yukawa-type [8]. We consider electron energy spectra for emission along the polarization axis in either direction, $\theta=0^{\circ}$ (left-hand detector, black curves) and $\theta$ $=180^{\circ}$ [right-hand detector, red (gray) curves]. Figure 1 illustrates the left-right asymmetry for a CE phase of $\phi=0^{\circ}$. The plots were generated by a purely numerical evaluation of

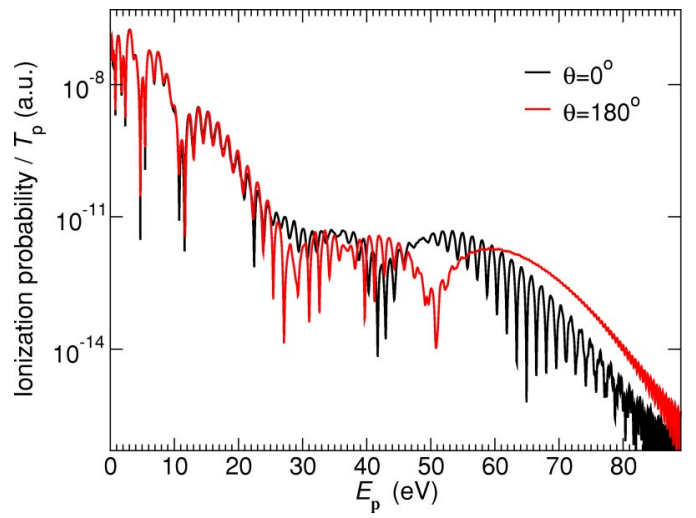

FIG. 1. (Color online) Differential ionization probabilities of $\operatorname{argon}\left(I_{p}=15.76 \mathrm{eV}\right)$ as functions of the electron energy $E_{\mathbf{p}}$, for emission in the direction $\theta=0^{\circ}$ (black curves) and $\theta=180^{\circ}$ [red (gray) curves], for the linearly polarized seven-cycle sine-square pulse (5) with CE phase $\phi=0$. The laser-field intensity is $10^{14} \mathrm{~W} \mathrm{~cm}^{-2}$ and the wavelength $800 \mathrm{~nm}$. The results were obtained by numerical integration, which is responsible for the jitter above $80 \mathrm{eV}$.

the matrix element $M_{\mathbf{p} 0}$ in the SFA, using methods introduced elsewhere [8] (these references also exhibit left-right spectra for $\mathrm{CE}$ phases other than $0^{\circ}$ ). One immediately realizes that the left-right asymmetry is much more pronounced for the rescattered than for the direct electrons $[5,13]$. For shorter pulses $\left(n_{p} \leqslant 6\right)$, this effect is bigger still. Much more eye-catching, however, is the presence of what appears like well-developed ATI peaks in one direction and their complete absence in the other. It is these structures that we will investigate in detail below.

The very high contrast of the ATI peaks calculated suggests that they are caused by interference of two or more contributions with almost equal magnitudes in the quantumorbit representation (1) of the transition amplitude. Therefore, in Fig. 2 we exhibit those quantum orbits that are instrumental in building up the spectra of Fig. 1. For $\theta=0^{\circ}$, the upper panel displays the contributions of the six most dominant orbits for $\theta=0^{\circ}$, which come in three pairs. As a general feature, the contributions of the two members of each pair differ for low electron energy. Then, at some well-defined energy, which almost agrees with the classical cutoff, they become nearly identical. For higher energy, the contribution of one of the two saddle points - the one drawn dashed in the figures - explodes exponentially and has to be dropped from the sum (1) [14]. The cutoff caused by the pair $\left(b, b^{\prime}\right)$ around $57 \mathrm{eV}$ matches the highest cutoff of the corresponding ATI spectrum in Fig. 1. The pair $\left(c, c^{\prime}\right)$ has a higher cutoff near $64 \mathrm{eV}$, but up to this point the contribution of $\left(b, b^{\prime}\right)$ is stronger by an order of magnitude. Around and beyond this cutoff, however, the orbits $b$ and $c$ make comparable contributions and, in consequence, the spectrum exhibits an extremely well-developed interference pattern. For an infinitely long pulse, $\left(c, c^{\prime}\right)$ and $\left(b, b^{\prime}\right)$ would be identical orbits, the latter being one period later than the former, so that only one would be included in the sum (4).

In the opposite direction $\left(\theta=180^{\circ}\right)$, the lower panel of Fig. 2 shows a very different picture. Many orbits contribute 

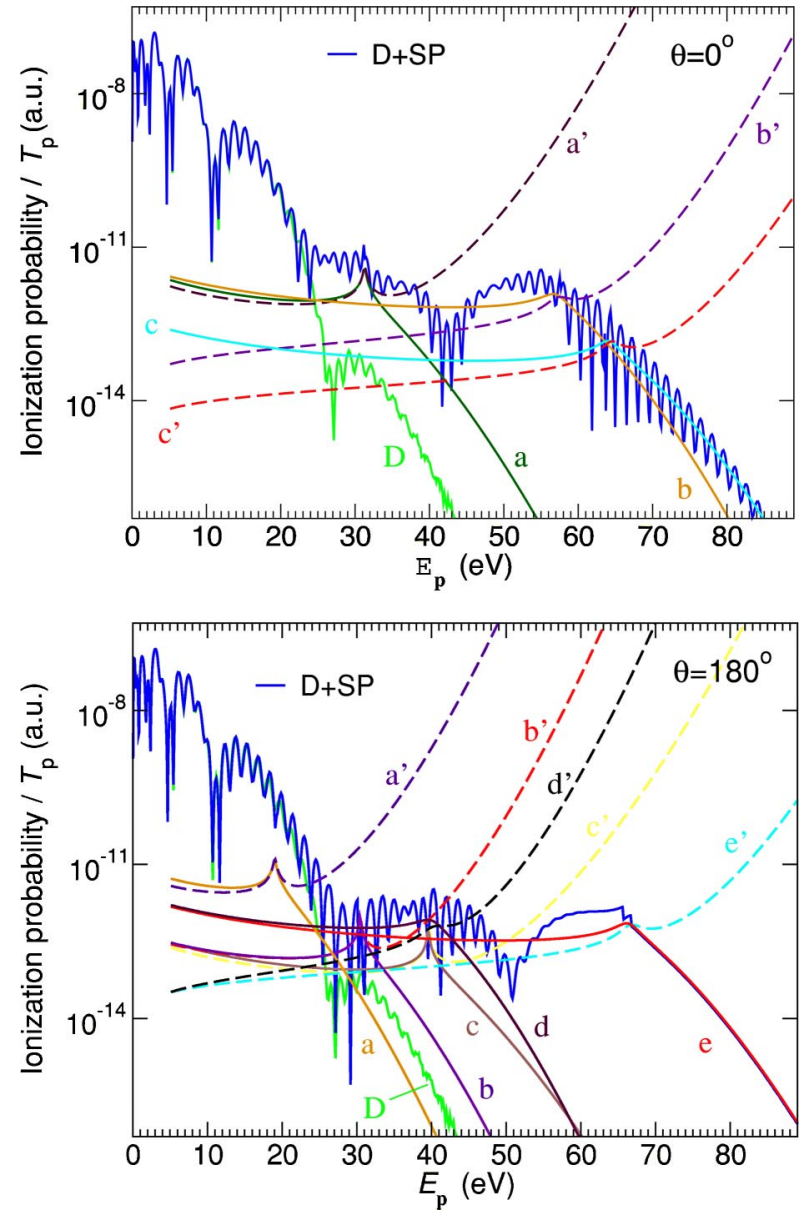

FIG. 2. (Color online) Quantum-orbit analysis of the spectra of Fig. 1. For $\theta=0^{\circ}$ (upper panel), the contributions of the dominant three pairs of quantum orbits are presented, as well as the directATI probability (D). The highly structured (dark blue) curve that extends from the upper left to the lower right gives the coherent sum of all these contributions (D+SP). For $\theta=180^{\circ}$ (lower panel), there are five dominant pairs as well as the direct electrons. Contributions represented by dashed lines must be excluded from the coherent sum (1) after their respective cutoffs.

to the medium-energy region, which is so complicated that a detailed analysis is futile. In contrast, the conditions at high energy are now completely different and very simple: only the pair $\left(e, e^{\prime}\right)$ contributes, which results in the complete absence of any interference pattern for energies above $55 \mathrm{eV}$.

The temporal evolution of the orbits (3) just discussed is depicted in Fig. 3, for the energy $56 \mathrm{eV}$. All orbits start at positions $\approx 0.6 A_{0} / \omega\left(A_{0}=E_{0} / \omega\right)$ on either side of the ion, which is close to the quasistatic "exit of the tunnel" at $I_{p} / E_{0}$. The origin of the nomenclature of the "long" and the "short orbit" becomes very apparent $[6,9]$. For $\theta=0^{\circ}$, the orbits $c$ and $c^{\prime}$ start at times when the field is weaker than at the later times when the orbits $b$ and $b^{\prime}$ take off. This is why the contribution of $c$ and $c^{\prime}$ to the sum (1) is less important except at high energies where this disadvantage is compensated by the fact that the field is stronger when they return and by the ensuing higher cutoff. Figure 3 also shows an orbit [orbit $a$; its partner $a^{\prime}$ is not shown since at the chosen energy of $56 \mathrm{eV}$ it is discarded from the sum (1)] that departs

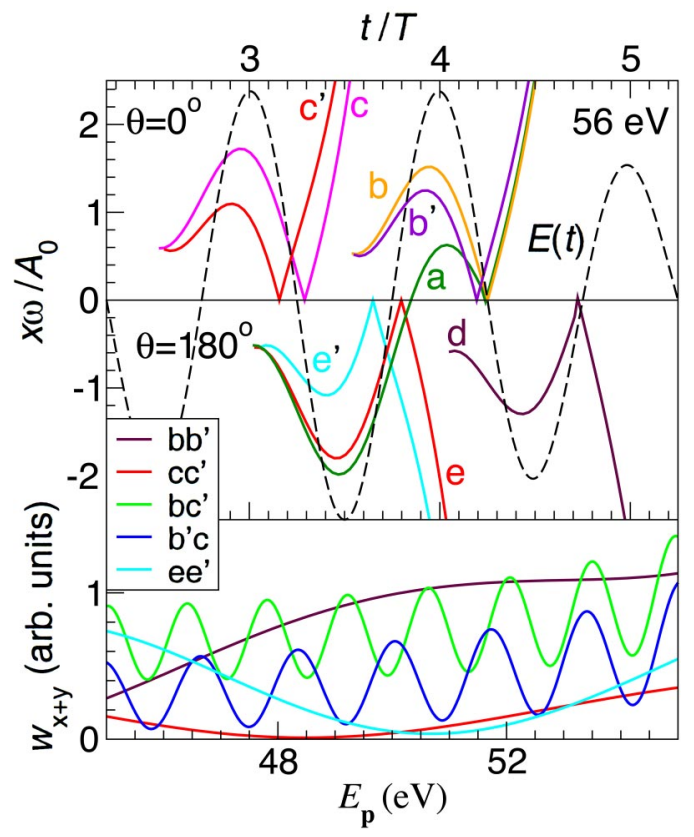

FIG. 3. (Color online) Real parts of the quantum orbits (3) investigated in Fig. 2, for the same parameters as in Fig. 1. Their distance from the ion (in multiples of the excursion amplitude $A_{0} / \omega$ ) is plotted as a function of time (in multiples of the carrierwave period $T$ ). The black dashed curve shows the seven-cycle electric field (5), which underlies the calculation. The upper and middle panels correspond to the directions $\theta=0^{\circ}$ and $\theta=180^{\circ}$, respectively. Only the orbits most important at $56 \mathrm{eV}$ are depicted. These are, using the nomenclature of Fig. 2, $a, b, b^{\prime}$, and $c, c^{\prime}$ for $\theta=0^{\circ}$, and $e, e^{\prime}$, and $d$ for $\theta=180^{\circ}$. The lowest panel displays the probabilities $w_{x+y}$ defined in the text for the orbits $x$ and $y$ specified in the inset.

from the ion in the direction opposite to the detector. Such orbits rescatter only upon their second revisiting the ion. Figure 2 shows that they only contribute to energies below about $35 \mathrm{eV}$. They are not responsible for the weak interference pattern between 25 and $40 \mathrm{eV}$ for $\theta=0^{\circ}$, since they return at the same time as the dominant orbits $b$ and $b^{\prime}$. This interference is rather caused by the weaker orbits $c$ and $c^{\prime}$. In the opposite direction $\left(\theta=180^{\circ}\right)$, as mentioned above only the orbits $e$ and $e^{\prime}$ contribute at high energy. They both return to the ion at about the same time right after the maximum of the field. Hence, they can gain an energy almost as high as in the long-pulse limit.

The lowest panel of Fig. 3 shows the spectrum that is generated when only two selected orbits, denoted by $x$ and $y$, are included in the coherent sum (1). We refer to the resulting probability by $w_{x+y}$. The figure shows that when the two orbits rescatter at about the same time, $w_{x+y}$ depends very smoothly on the energy $E_{\mathbf{p}}$; cf. the orbits $(x, y)=\left(b, b^{\prime}\right)$, $\left(c, c^{\prime}\right)$, and $\left(e, e^{\prime}\right)$. If, on the other hand, their return times $t_{1 x}$ and $t_{1 y}$ are about one cycle apart, $w_{x+y}$ displays a fringe pattern with a peak separation of about $\omega$. More precisely, if $\Delta t=\left|t_{1 x}-t_{1 y}\right|<T$, we observe a fringe separation of more than $\omega$ [in the case where $\left.(x, y)=\left(b^{\prime}, c\right)\right]$, and vice versa if $\Delta t>T\left[\right.$ in the case where $\left.(x, y)=\left(b, c^{\prime}\right)\right]$.

This behavior can be traced back to the action (2). For the 
interference pattern, only the final part of the orbit matters, which starts at the time $t_{1 s}$ when the electron finally leaves the ion towards the detector. Given two such orbits $x$ and $y$, by evaluating their actions $S_{x}(\mathbf{p})$ and $S_{y}(\mathbf{p})$ one may convince oneself that their superposition yields the transition probability

$$
w_{x+y}\left(E_{\mathbf{p}}\right) \propto\left|M_{\mathbf{p} 0}\right|^{2} \propto \alpha+\beta \sin \left(E_{\mathbf{p}} \Delta t+\psi\right),
$$

where the parameters $\alpha, \beta$, and $\psi$ are functions of the coefficients $a_{x}$ and $a_{y}$ in Eq. (1). This expression holds provided $\int_{t_{1 y}}^{t_{1 x}} d \tau \mathbf{A}(\tau)$ can be neglected, which is usually the case, because $\Delta t$ is either small or $\approx T$. The position of the maxima depends on the parameter $\psi$. The fringe separation is $\Delta E_{\mathbf{p}} / \omega=T / \Delta t$.

While, in Fig. 3, the contributions of selected orbits such as $\left(b, c^{\prime}\right)$ and $\left(b^{\prime}, c\right)$ display a fringe separation $\Delta E_{\mathbf{p}} \neq \omega$, the total transition probability summed over all pairs of orbits shows almost exactly $\Delta E_{\mathbf{p}}=\omega$. This will be different as soon as the atomic dynamics deal differently with the long and the short orbit of a pair. A real many-electron atom will see to that, via its particular effective binding potential, and a mol- ecule even more so. In consequence, analyzing the fringe separation in the high-energy ATI spectrum allows one to deduce very subtle details of the ionization dynamics on a time scale of a small fraction of the optical cycle, without having to utilize an additional attosecond pulse [15]. However, interpreting the fringe pattern will not be easy.

In summary, we have extended the formalism of quantum orbits to the case of high-order above-threshold ionization generated by few-cycle laser pulses. Thereby, the many features of spectra that develop upon variation of the CE phase and the pulse duration can be explained in terms of the properties of a few quantum orbits, which are closely related to classical electron trajectories. In particular, we have shown that the interference patterns of few-cycle ATI spectra can be controlled by the $\mathrm{CE}$ phase. Conversely, such patterns can reveal subtle features of the ionization dynamics deep down into the attosecond time scale.

We are grateful to P. Agostini for discussions. This work was supported in part by VolkswagenStiftung and by the Federal Ministry of Education and Science, Bosnia and Herzegovina.
[1] T. Brabec and F. Krausz, Rev. Mod. Phys. 72, 545 (2000); S. T. Cundiff, J. Phys. D 35, R43 (2002); P. Agostini and L. F. DiMauro, Rep. Prog. Phys. 67, 813 (2004).

[2] G. G. Paulus, F. Grasbon, H. Walther, P. Villoresi, M. Nisoli, S. Stagira, E. Priori, and S. De Silvestri, Nature (London) 414, 182 (2001).

[3] A. Baltuška, Th. Udem, M. Uiberacker, M. Hentschel, E. Goulielmakis, Ch. Gohle, R. Holzwarth, V. S. Yakovlev, A. Scrinzi, T. W. Hänsch, and F. Krausz, Nature (London) 421, 611 (2003).

[4] M. Nisoli, G. Sansone, S. Stagira, S. De Silvestri, C. Vozzi, M. Pascolini, L. Poletto, P. Villoresi, and G. Tondello, Phys. Rev. Lett. 91, 213905 (2003).

[5] G. G. Paulus, F. Lindner, H. Walther, A. Baltuška, E. Goulielmakis, M. Lezius, and F. Krausz, Phys. Rev. Lett. 91, 253004 (2003).

[6] G. Sansone, C. Vozzi, S. Stagira, M. Pascolini, L. Poletto, P. Villoresi, G. Tondello, S. De Silvestri, and M. Nisoli, Phys. Rev. Lett. 92, 113904 (2004); G. Sansone, C. Vozzi, S. Stagira, and M. Nisoli, Phys. Rev. A 70, 013411 (2004).

[7] P. B. Corkum, Phys. Rev. Lett. 71, 1994 (1993).

[8] D. B. Milošević, G. G. Paulus, and W. Becker, Opt. Express 11, 1418 (2003); Laser Phys. Lett. 1, 93 (2004).

[9] M. Lewenstein, Ph. Balcou, M. Yu. Ivanov, A. L'Huillier, and
P. B. Corkum, Phys. Rev. A 49, 2117 (1994).

[10] R. Kopold, D. B. Milošević, and W. Becker, Phys. Rev. Lett. 84, 3831 (2000).

[11] P. Salières, B. Carré, L. Le Déroff, F. Grasbon, G. G. Paulus, H. Walther, R. Kopold, W. Becker, D. B. Milošević, A. Sanpera, and M. Lewenstein, Science 292, 902 (2001).

[12] W. Becker, F. Grasbon, R. Kopold, D. B. Milošević, G. G. Paulus, and H. Walther, Adv. At., Mol., Opt. Phys. 48, 35 (2002).

[13] S. Chelkowski, A. D. Bandrauk, and A. Apolonski, Phys. Rev. A 70, 013815 (2004); S. X. Hu and A. F. Starace, ibid. 68, 043407 (2003).

[14] If the uniform approximation rather than the saddle-point approximation is used, there is no need to discard one solution by hand: C. Figueira de Morisson Faria, H. Schomerus, and W. Becker, Phys. Rev. A 66, 043413 (2002).

[15] R. Kienberger, E. Goulielmakis, M. Uiberacker, A. Baltuska, V. Yakovlev, F. Bammer, A. Scrinzi, Th. Westerwalbesloh, U. Kleineberg, U. Heinzmann, M. Drescher, and F. Krausz, Nature (London) 427, 817 (2004); E. Goulielmakis, M. Uiberacker, R. Kienberger, A. Baltuska, V. Yakovlev, A. Scrinzi, Th. Westerwalbesloh, U. Kleineberg, U. Heinzmann, M. Drescher, and F. Krausz, Science 305, 1267 (2004). 